\title{
Development and application of a flexible light sheet fluorescence microscope for high speed 2D and 3D imaging of calcium dynamics in cardiomyocytes.
}

Liuba Dvinskikh, Hugh Sparks, Alice Francis, Carl Paterson, Sian Harding, Ken MacLeod, Chris Dunsby Imperial College London, United Kingdom

\section{Abstract Text}

The high prevalence and poor prognosis of heart failure are two key scientific drivers behind research into the electrophysiology of healthy and damaged cardiac tissue. Dyssynchronous calcium release and disorganization in the t-tubule structure within individual cardiomyocytes has been linked to poor contractile function and arrhythmia. Confocal line scan microscopy has been used widely for single dimensional analysis of calcium signals in cardiomyocytes. However, the physiological anisotropy of cardiomyocytes is fundamental to calcium signalling and impulse propagation through adjacent cells in heart tissue and the extension of confocal scanning to more spatial dimensions comes at the cost of reduced signal to noise ratio and temporal resolution.

Correlative imaging of the calcium dynamics of cardiomyocytes and their microstructure calls for the use of imaging techniques capable of high-speed two-dimensional or real-time video-rate threedimensional microscopy of cardiac tissue at subcellular resolution. This work implements a custom-developed inverted light sheet fluorescence microscope for studying calcium dynamics in cardiomyocytes [1]. The system can operate using two different illumination modes: slower high-resolution imaging using scanned line illumination, and light sheet illumination for fast high-speed 2D and 3D imaging with lower phototoxicity and photodamage to the cells. Video rate volumetric acquisition is achieved by scanning the detection focal plane using folded remote refocusing [2], scanned synchronously with the axially-swept illumination light sheet, with 
minimal mechanical disturbance of the sample.

The spatial and temporal resolution achieved allows

multidimensional characterization of calcium dynamics across single cardiomyocytes in correlation with t-tubule microstructure. We present data obtained with this system demonstrating twodimensional dual channel optically sectioned time-lapse imaging of calcium dynamics in isolated live rat cardiomyocytes at $150 \mathrm{fps}$ and $0.5 \mu \mathrm{m}$ lateral resolution. Electrical pacing is used to stimulate calcium transients with decoupled contraction. The fluorescence intensity trace of calcium indicator Fluo-4 is used to characterize the spatial variation in transient rise time across the cell (Fig. 1). Preliminary results indicate some variation in the time to half maximum within a cell, which was seen to be consistent across multiple transients within the same cell.

(a)

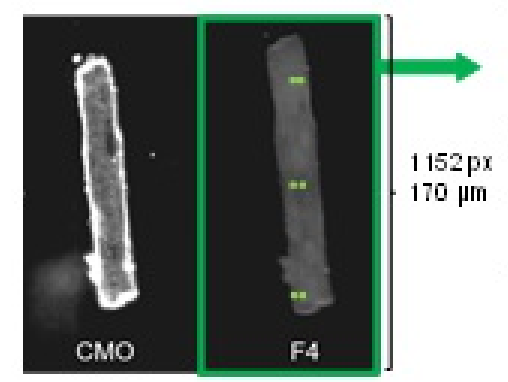

(b)

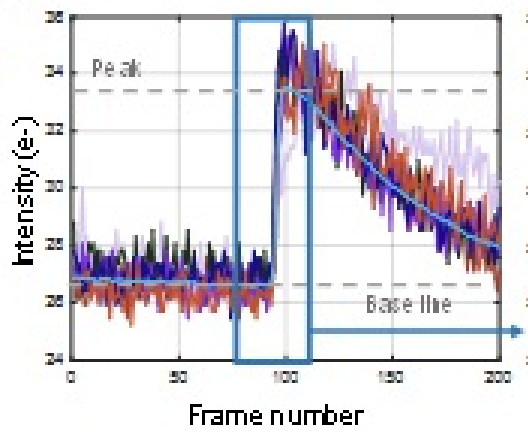

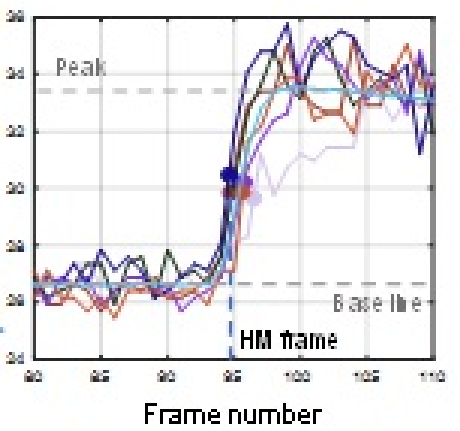

(c)

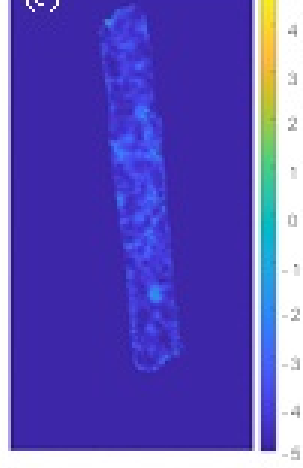

Relative Hov tame

Fig. 1 Calcium transient analysis in live cardiomyocytes (a) Spatially separated Cell Mask Orange (CMO) and Fluo-4 (F4) emission at peak of $\mathrm{Ca}^{2+}$ transient. Intensity traces of pixels at indicated positions (green dots) are shown in (b): Fluo-4 emission intensity cell average (bold) and example individual pixel traces with linearly interpolated half-maximum (HM) frames. (c) Variation of relative frame number $(6.67 \mathrm{~ms} /$ frame) corresponding to the half-maximum across cell for an individual transient. Image dimensions: $85 \mu \mathrm{m} \times 170 \mu \mathrm{m}$. 